of an acrylic monomer (isobornyl acrylate); one is dispersed with carbon black pigments and another with titanium oxide white pigments. The two solutions are fed into each arm of the $\mathrm{Y}$, respectively, to form a two-color stream at the junction. The two-color stream travels down the leg of the $\mathrm{Y}$ and forms droplets when it meets the co-flowing stream of a poly(vinyl alcohol) solution. The droplets are then carried by the co-flowing stream and polymerized into solid microscopic particles outside the fluidic module. The researchers showed that droplets of uniform size could be produced under a limited range of precisely controlled fluidic conditions. Well-defined biphasic particles $117 \mu \mathrm{m}$ in diameter were formed in these experiments. The researchers also determined that smaller particles (20-30 $\mu \mathrm{m}$ in diameter), which would be needed for high-resolution displays, could be formed, although with a less well-defined two-color boundary.

The prepared microparticles are responsive to electric fields. The researchers built a flat-panel display device with the microparticles dispersed between two electrode panels. In the presence of an external electric field, these microspheres turned to orient their black half to the negatively charged panel. When the researchers reversed the electric field, the particles flipped.

The researchers also showed that it would be possible to scale up the manufacture of Janus particles for commercial applications. They further said that the technique is not limited to polymer components; ceramic or metallic materials are also possible. The researchers speculated that spheres with magnetic anisotropy could be formed by dispersing magnetite in one hemisphere, and that spheres with optical anisotropy could be formed by incorporating a liquid-crystal phase. They also pointed toward the use of "barcoded" particles in potential applications such as "biological multiplexing, multicomponent carriers for targeted drug delivery, and 3D photonic crystals of different domains."

SHIMING WU

\section{Self-Assembled CNT-Based Electronic Devices}

Despite significant progress in the scientific understanding of the electronic, optical, and mechanical properties of carbon nanotubes (CNTs), large-scale technological applications of CNTs remain rare. This is partly due to the fact that controlled and efficient assembly of CNTs into electronic devices with high spatial precision remains a major challenge. Now, C. Klinke, J.B. Hannon, A. Afzali, and P. Avouris from the IBM T.J. Watson Research Center in New York have discovered that the use of chemical functionalizing could become an efficient approach to the creation of commercial-scale applications for selfassembled CNT-based electronic devices.

Most CNT devices have been fabricated through electron-beam lithography. Commercial production, however, poses challenges, since this lithography technique cannot be applied at large production scales because of its small yield.

Klinke and co-workers-as reported in the May issue of Nano Letters (p. 906; DOI: 10.1021/nl052473f)—used a high-dielectric- constant insulator as the substrate on which to assemble their devices. The unique electrical properties of nanotubes, however, are destroyed when another chemical species is covalently attached to them. To overcome this obstacle, the researchers used a reversible functionalization of nanotubes with a chemical group that makes the CNTs likely to bind specifically to certain materials such as hafnium dioxide or aluminum oxide-all basic high-к dielectrics - but not to silicon oxide. Once the CNTs have attached themselves at the desired location, the chemical group is removed by a thermal annealing process.

Selective attachment was achieved using the following procedure. Long and narrow aluminum films were first deposited on a $\mathrm{SiO}_{2}$ substrate. $\mathrm{Al}_{2} \mathrm{O}_{3}$ was created by oxidizing the $\mathrm{Al}$ films using an oxygen plasma. The CNTs were functionalized with a diazonium salt and dispersed in either methanol or ethanol, which was then deposited as a liquid film on the substrate. The liquid film was dried on a hot plate, sonicated in pure methanol, and then dried in $\mathrm{N}_{2}$. The CNTs aligned along the narrow $\mathrm{Al}_{2} \mathrm{O}_{3}$ strips. Field-effect transistors were constructed by depositing Pd to produce source and drain structures.

After this processing, the resulting transistors demonstrated excellent electrical properties. This new technique provides the possibility for large-scale integration of CNT-based electronic components into electronic devices, the researchers said.

MARKUS J. BUEHLER

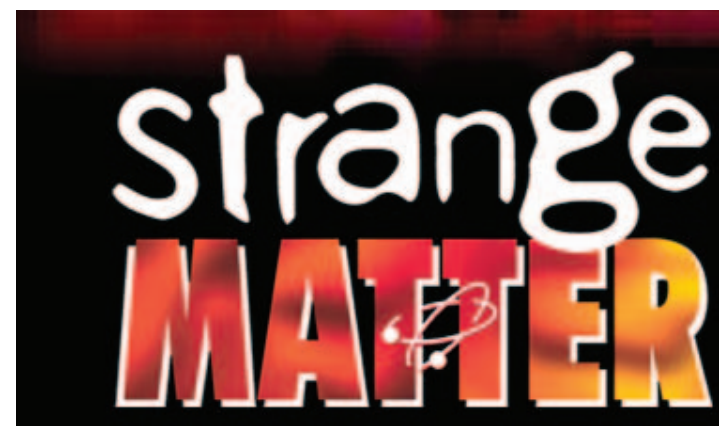

Strange Matter is presented by the Materials Research Society.

This exhibition and its tour are made possible by the generous support of the National Science Foundation, Alcan Inc., Dow,

Ford Motor Company Fund, Intel Innovation In Education, and the $3 M$ Foundation.

\section{Experience this interactive materials science exhibition}

Telus World of Science, Edmonton, A/berta May - September 2006

Da Vinci Discovery Center of Science and Technology, Allentown, PA May - September 2006

Space Center Houston, Houston, TX October - December 2006

To volunteer for activities with the exhibition, contact Amy Moll

Community Resources Coordinator at amoll@boisestate.edu
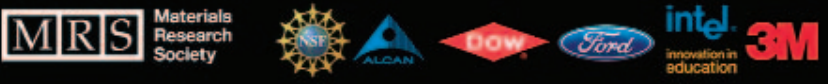